\title{
Cutaneous leishmaniasis at Wasit governorate
}

\author{
Abdulsadah A.Rahi* \\ Received 25, September, 2009 \\ Accepted 30, May, 2010
}

\begin{abstract}
:
The study included identification of Leishmania parasites from cases of cutaneous leishmaniasis, by examination of smears for LD bodies, isolation of parasites by culture in media, and characterisation of the isolated parasites by Isoenzyme analysis.

Out of 100 cases suspected for cutaneous leishmaniasis ; 85 were diagnosed on culture for Leishmania promastigotes and this method was found to be superior to direct microscopy for amastgotes (LD bodies).The cases included in this study belonged to different areas of Wasit/Iraq. There were 30 cases from Hay, 35 from Badra, and 35 cases from Suwaira.The distribution of infection in different age groups indicated that majority of cases belonged to young and middle aged adults. The infection was detected in both sexes with a predominance in males. The clinical picture of cutaneous lesions was suggessive of both wet and dry types of lesions.
\end{abstract}

Key words: ( Cutaneous leishmaniasis , Leishmania , Isoenzyme)

\section{Introduction:}

Leishmaniasis, a vector - borne disease caused by obligate intramacrophage protozoa, is characterized by diversity and complexity [1]. A total of about 21 Leishmania species have been identified to be pathogenic to human. Leishmania are one of several genera within the family Trypanosomatidae, and are characterized by the possession of a kinetoplast, a unique form of mitochondrial DNA. Leishmaniasis is endemic in 88 countries of 5 continents with a total of 350 people risk and 12 million cases with estimated incidence of 1- 1.5 million cases of cutaneous leishmaniasis (CL)and 500,000 cases of visceral leishmaniasis (VL) [1].

Leishmaniasis is caused by different species of Leishmania, under kingdom Prostista and phylum
Euglenozoa [2]. The parasite Leishmania exists at least in two forms [3]:

1-Amastigote form: Amastigotes are ovoid and non flagellated form of Leishmania , measuring 3-5 $\mu \mathrm{m}$ in length.

2-Promastigote form: Flagellated form found in the sandfly host .

Worldwide,vector-borne

transmission is the most common mode of transmission. Other modes of transmission such as parenteral, congenital, sexual,occupational exposures, and person to-person transmission could also theoretically occur [4].

\section{Materials and Methods:}

Sample collection and culture:-

One hundred samples from patients with skin lesion suspected of cutaneous leishmaniasis (CL) were

\footnotetext{
*Wasit university/College of Science
} 
randomly collected from different endemic areas of Wasit province / Iraq. The samples were aspirated from the edges of the skin lesions and cultured on Novy-MacNeal-Nicole (NNN) media. Eighty-five isolates growing in the culture were used for characterization.For isoenzyme analysis,primary isolates were subcultured on (NNN) media, supplemented with $2 \mathrm{mM}$ L-glutamine, $15 \%$ fetal bovine serum, $100 \mathrm{U} / \mathrm{ml}$ penicillin \&100 $\mu \mathrm{g} / \mathrm{ml}$ streptomycin. The second or third subcultures were used for characterization.

\section{Isoenzyme electrophoresis [5]:-}

Discontinuous vertical PAGE and cellulose acetate were used for isoenzyme analysis of the isolates. Promastigotes were harvested at the end of logarithmic phase by centrifugation at $3000 \times \mathrm{g}$ at $4^{\circ} \mathrm{C}$ for 20 min. The supernatants were discarded and the pellets of promastigotes were washed three times by $\mathrm{PBS}(\mathrm{pH}$ 7.2). The pellets of promastigotes were mixed with equal volumes of a hypotonic aqueous solution of enzyme stabilizer (1mM EDTA, 1 mM @amino -n- caproic acid, $1 \mathrm{mM}$ dithiothreitol), frozen by placing in vapor phase of liquid nitrogen and thawed at $25-30^{\circ} \mathrm{C}$ for three times. Soluble extract of lysed promastigotes was prepared by centrifugation at $30,000 \times \mathrm{g}$ at $4^{\circ} \mathrm{C}$ for $30 \mathrm{~min}$, and stored at $-70^{\circ} \mathrm{C}$ until use .

\section{Results and Discussions:}

Out of 85 cases were diagnosed for cutaneous leishmaniasis on culture of exudates from lesions. Table (1) shows the positive cases of direct microscopy $(60 \%)$, culture on $\mathrm{NNN}$ media $(85 \%)$ and analysis of isoenzyme $(100 \%)$ for cutaneous leishmaniasis. These results showed higher sensitivity and specificity of isoenzyme analysis than other methods in identification of Leishmania species and with agreement of results were recorded by [6]. Table( 2)shows the distribution of cases of cutaneous leishmaniasis in different age groups and divided into wet and dry lesions. It was found that the majority of cases between (13-60) years and the wet lesion was predominance. The results are similar to other studies carried out in Iraq [7], and in other countries [8]. The isoenzyme profile of 100 cases of cutaneous leishmaniasis recovered in present study showed two distinct patterns $[9,10]:-$

1- Banding pattern identical to that obtained with the refernce strain of Leishmania major.

2- Banding pattern identical to the refernce strain of Leishmania tropica.

Table(1)Results of microscopy,culture \& isoenzyme for Leishmania

\begin{tabular}{|c|c|c|c|}
\hline Total suspected $\quad$ cases of CL & +Ve for LD by direct microscopy & +Ve for culture on NNN media & $\begin{array}{c}\text { +Ve for analysis } \\
\text { of isoenzyme }\end{array}$ \\
\hline 100 & $60(60 \%)$ & $85(85 \%)$ & $100(100 \%)$ \\
\hline
\end{tabular}

Table(2) Distribution of cases of Cutaneous Leishmaniasis in different age groups

\begin{tabular}{|c|c|c|c|}
\hline Age groups & +ve cases & +ve cases with wet lesions & +ve cases with dry lesions \\
\hline Children (1-12)years & $15(15 \%)$ & $10(10 \%)$ & $\mathbf{5 ( 5 \% )}$ \\
\hline Young adults (13-30)years & $35(35 \%)$ & $20(20 \%)$ & $15(15 \%)$ \\
\hline Adults (31-60)years & $40(40 \%)$ & $\mathbf{2 8}(28 \%)$ & $\mathbf{1 2 ( 1 2 \% )}$ \\
\hline Old (above 60 years) & $10(10 \%)$ & $\mathbf{6 ( 6 \% )}$ & $\mathbf{4 ( 4 \% )}$ \\
\hline Total +Ve cases & $100(100 \%)$ & $\mathbf{6 4}(64 \%)$ & $\mathbf{3 6}(36 \%)$ \\
\hline
\end{tabular}




\section{References:}

1. Desjeux, P. 2001. The increase in risk factors for leishmaniasis worldwide. Trans. R. Soc. Trop.Med. Hyg., 95(2):239-243.

2.Lainson R. and Shaw JJ. 1987. Evolution, classification and geographi-cal distribution. The leishmaniases in biology and medicine. London: Academic Press; p. 1-120.

3. Ross R. 1993.Note on the bodies recently described by Leishman and Donovan. Br Med J ; 2(1) : 1261-2.

4.Ashford RW and Bates PA. 1998. Leishmaniasis in the Old World. Vol.5. Parasitology. 9th ed.,USA: Oxford University Press; p. 215-40.

5. Piarroux, R.Trouvé,V. Pratlong,F. Martini,A. and Rioux, J. 1994.The use of isoelectric focusing on polyacrylamide gel for the enzymatic analysis of Old World Leishmania species.Trans. R. Soc. Trop. Med. Hyg.,88 (5):475-478.
6. Lee S T, Chiang S C, Singh A K, Liu H Y. 2002. Identification of Leishmania species by a specific DNA probe that is conserved only in the maxicircle DNA of humaninfective Leishmania. J Inf. Dis 172 (3):891-894.

7. Korzeniewski, K. 2005. Health hazards in Iraq, Lekarz Wojskowy, 81(3): 176-180.

8.Singh, S. and Sivakumar, R. 2003. Recent advances in the diagnosis of leishmaniasis. J .Postgrad. Med., 49(5) : 55-60.

9. Markle,W H. and Makhoul, K. 2004. Cutaneous leishmaniasis: recognition and treatment. Am. Fam. Physician. 69 : 1455-1460.

10.KumarV, Kishore K,Palit A,Sharma MC,and Das VN. 2001. Vectorial efficacy of Phlebotomus argentipes in Kala - azar endemic foci of Bihar under natural and artificial conditions. J Comm Dis; 33(2) : 102-9.

\section{اللشمانيا الجلدية في محافظة واسط}

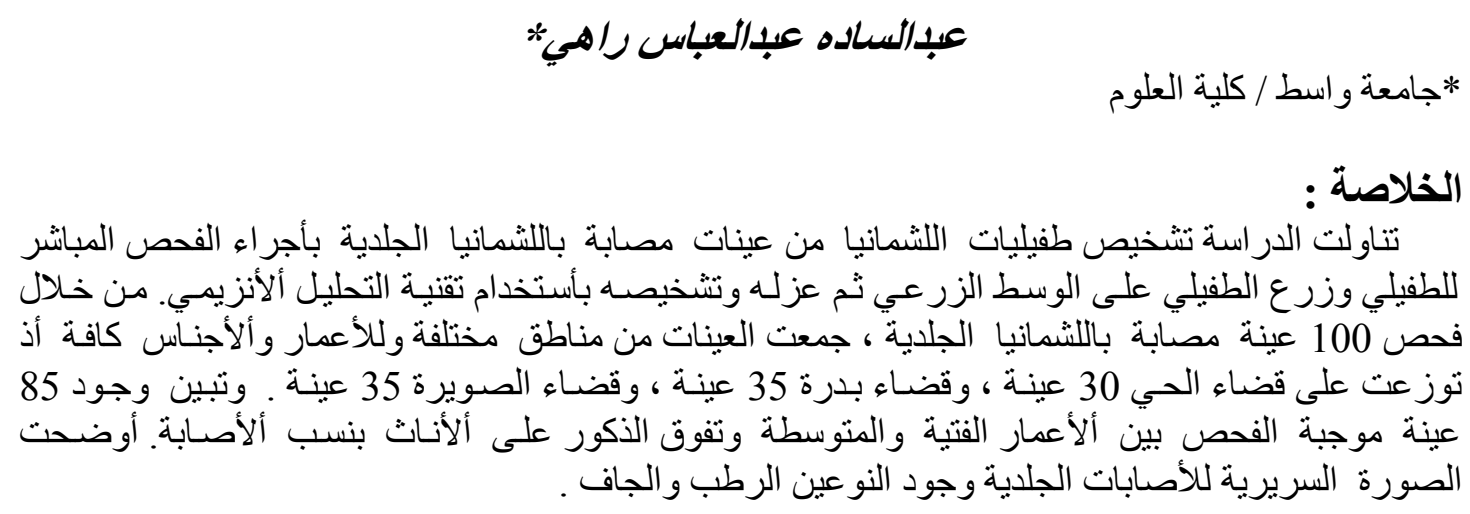

